\title{
INTEGRACIÓN TERRITORIAL DE PALESTINA CALDAS \\ A TRAVÉS DE LOS MACROPROYECTOS DE IMPORTANCIA REGIONAL ${ }^{1}$
}

\author{
NORBEY CASTRO $\mathrm{GIL}^{2}$ \\ CARLOS ANDRES LOPEZ GIRALDO ${ }^{3}$
}

\begin{abstract}
Recibido el 4 de mayo de 2012 y aprobado el 21 de agosto de 2012
\end{abstract}

\section{RESUMEN}

El desarrollo de estrategias y mecanismos para la integración territorial es de gran importancia para el municipio de Palestina y otros similares que ostentan la categoría más baja dentro de la clasificación municipal, condicionada por algunas características de desarticulación física y funcional en el territorio. Asimismo, es relevante y extrapolable a otros municipios que han sido objeto de la implantación de macroproyectos de importancia regional, sin la planeación adecuada, ni una articulación al contexto y a las características municipales.

La primera contribución de esta investigación consiste en la elaboración de la caracterización regional del territorio y de la unidad de análisis, desde las dimensiones del desarrollo y sus atributos, estableciendo los desequilibrios en la ocupación del territorio, pero también sus potencialidades y oportunidades de desarrollo.

Posteriormente se establecen los escenarios prospectivos posibles para el territorio analizado, desde la espacialización de los determinantes de ordenamiento territorial que en la actualidad orientan los usos del suelo y su confrontación con las perspectivas generales de desarrollo y se determinan los escenarios propuestos, los cuales simulan una forma de ocupación de las áreas de expansión de la microrregión, considerando las oportunidades, posibilidades y limitantes que genera el establecimiento de los macroproyectos.

Seguidamente se establecen las líneas de acción derivadas de los escenarios prospectivos y la construcción de los macroproyectos con incidencia subregional, micro-regional y municipal, en los modelos de ocupación territoriales, desequilibrios regionales, usos del suelo, conectividad vial, reconversión de usos y solución de déficits, recuperación de áreas ambientalmente sensibles, relocalización de asentamientos y en la articulación modal, entre otros factores. 
Por último se formulan los lineamientos de política y objetivos que permitirán el desarrollo de un proyecto de ciudad, dirigidos a influir profundamente en la red urbano regional, promoviendo la adopción de los modelos de desarrollo sostenible, endógeno, local y regional, enfoques que originen de manera específica y en forma complementaria, modelos centrados en logros cualitativos como la urbanización compacta, variada, multipolar, equilibrada, limpia y eficiente energéticamente.

\title{
PALABRAS CLAVE:
}

Integración, macroproyectos, desarrollo, prospectiva, ordenamiento.

\section{TERRITORIAL INTEGRATION IN PALESTINA, CALDAS TRHOUGH MACROPROJECTS OF REGIONAL IMPORTANCE}

\begin{abstract}
Developing strategies and mechanisms for regional integration is of great importance to the municipality of Palestina and other similar municipalities which hold the lowest category in the municipal influenced classification becaue of some physical and functional disruption characteristics in the territory. It is also relevant and applicable to other municipalities where large scale projects of regional importance, have been implementing without either proper planning or an articulation with the context and municipal characteristics.
\end{abstract}

The first contribution of this research is the development of the regional characterization of the territory and the unit of analysis, from the dimensions of development and its attributes, and setting the imbalances in land use, but also its potential and development opportunities.

Subsequently, the possible prospective scenarios for the analyzed area are established from the spatial determinants of land that currently guide land use and its confrontation with the overall development perspectives and the proposed scenarios are determined, which simulate a form of occupation of the mirco-region expansion areas, considering the opportunities, possibilities and constraints generated by the establishment of the macro projects.

Afterwards, the lines of action arising from the prospective scenarios and the construction of the macroprojects with a sub-regional, microregional and municipal impact in the models of territorial occupation, regional imbalance, land 
use, road connectivity, use conversion and deficits solution, recovery of environmentally sensitive areas, relocation of settlements and modal articulation, among other factors, are established.

Finally, the policy guidelines and objectives which allow the development of a city project are formulated, directed to influence profoundly the regional urban network, promoting the adoption of sustainable development models, endogenous, local and regional approaches that originate in an specific and complementary way originate, qualitative achievement centered models such as, varied, multi-polar, balanced, clean and energy efficient compact urbanization.

\section{KEY WORDS}

Integration, macroprojects, development, prospective, ordering.

\section{INTRODUCCIÓN}

La desarticulación de los sectores suburbanos de Arauca y Santágueda, del municipio de Palestina, y de los municipios de la sub-región Centro Sur, con sus previsibles consecuencias en las dimensiones del desarrollo y sus atributos, cobra actualmente una mayor importancia en razón del establecimiento de los macroproyectos, Aeropuerto del Café, Ferrocarril de Occidente y Plan Vial Centro-Sur en su territorio. Estos hechos han obligado a un replanteamiento del ordenamiento territorial del municipio y la subregión, para potenciar sus posibilidades de inserción en los mercados globales, aprovechando las ventajas comparativas de localización en el contexto nacional, la competitividad de sus mercados y las evidencias que se tienen en el sentido, que la reestructuración capitalista y la globalización, están convirtiendo las grandes áreas urbanas en las formas espaciales dominantes de la nueva geoeconomía mundial.

La re-significación y recuperación del rol protagónico del componente territorial como espacio de especificidad e identificación, obliga a repensar las ciudades como actores dentro de una región y mirar más allá de sus límites; de este modo es indispensable concebir e implementar una nueva generación de políticas regionales, como consecuencia de los efectos funcionales y espaciales de la globalización y estrategias endógenas de desarrollo para adaptarse a sus cambios. 
Estos desafíos de las ciudades implican organizar su proceso de ascenso y crear la institucionalidad apropiada para su desarrollo e integración territorial, como resultado de la concertación estratégica entre estado, mercado y sociedad civil local. El desarrollo local sostenible y la democracia participativa son las condiciones insustituibles de los nuevos modelos de desarrollo a escala humana que se perfilan en el mundo, basados en las condiciones particulares de cada territorio y en las potenciales, que lo pueden hacer competitivo frente a otras economías territoriales.

Dicho ejercicio pretende generar conocimiento sobre el territorio estudiado y proporciona una herramienta de análisis de la manera como se construyen modelos de ocupación que producen "territorios ganadores", conduciendo a una diferenciación entre ellos. A partir del conocimiento del modelo de ocupación territorial actual del municipio de Palestina y teniendo en cuenta las dimensiones del desarrollo, su estructura, desequilibrios del territorio, articulación, problemas, potencialidades y restricciones, en una perspectiva de sostenibilidad, se construirá una propuesta de modelo de ocupación futuro del territorio, que pretende ser un punto de partida para las decisiones a futuro. Tal modelo sintetiza los principales postulados, principios y propuestas para el modelo de desarrollo sostenible, articulados a las condiciones particulares del área de análisis. El modelo propuesto, sometido a la discusión y consenso entre los actores regionales, posibilitará la construcción colectiva de futuro en la zona al generar una serie de recomendaciones y sugerencias que tendrán gran valor en la formulación del modelo territorial elegido.

El aporte de la investigación sobre la integración territorial de Palestina y la generación de nuevo conocimiento sobre el tema, se basa en dos (2) premisas: la primera, la condición especial del territorio, favorecido sectorial y espacialmente por proyectos relacionados con la movilidad regional; de esta manera el territorio de análisis obtiene una ventaja comparativa en cuanto a su posición relativa y el trabajo de investigación se constituye en una herramienta que muestra los lineamientos de manejo de situaciones que reflejan la ausencia de planeación en la concepción de tales macroproyectos, los cuales impactan abruptamente los municipios sin permitirles una preparación homeostática para su incorporación al territorio. La segunda incluye las propuestas de complementación del plan básico de ordenamiento de Palestina, el cual adolece de instrumentos que propicien el desarrollo de sus áreas suburbanas y rurales, condición generalizada en el país para estas 
herramientas de gestión territorial, que no permite considerar su verdadera dimensión y valor.

No existen ejercicios de planificación similares con base en experiencias de municipios que como Palestina se ven impactados por proyectos considerados como prioridades regionales, que desarticulan y reorientan el ordenamiento existente; tampoco sobre modelos de integración intermunicipal o inter-regional, propiciada por macro proyectos planificados o no, por lo tanto el objetivo general de identificar estrategias y proponer mecanismos de integración territorial, a través del análisis de las dimensiones del desarrollo del territorio de Palestina, implica la creación de un camino conceptual y metodológico que corresponda con la realidad territorial analizada, además de responder la pregunta ¿Cómo generar procesos de integración territorial en el municipio de Palestina, Caldas?

Palestina y en general la subregión centro-sur de Caldas se localizan en la zona geográfica tres (3) de la Cordillera Central en la ecorregión Eje Cafetero, de la que hacen parte además, Pereira, Armenia, Manizales y sus zonas con procesos de metropolización. Se trata de municipios de pie de monte, ubicados entre las cotas 1200 y 1800 msnm. Es en esta zona de economía cafetera donde se concentra el mayor desarrollo urbano de la ecorregión, con ciudades prestadoras de servicios secundarios y terciarios, que tienen fuertes relaciones funcionales entre ellas como vínculos de tipo económico y cultural.

La subregión Centro-Sur está conformada por los municipios de Manizales, Chinchiná, Neira, Palestina y Villamaría; se localiza al sur del departamento de Caldas y limita por el norte con los municipios de Aranzazu y Filadelfia, por el oriente con Marulanda, por el sur con el departamento de Risaralda y por el occidente con los municipios de San José, Risaralda y Anserma.

El cultivo del café en el viejo Caldas y especialmente en la subregión centro-sur del departamento, no sólo ha sido una actividad económica generadora de ingresos: el desarrollo económico y social del departamento, al igual que el de los municipios que conforman el llamado Eje Cafetero Colombiano, históricamente ha estado ligado a la evolución de la caficultura, de tal forma que la apropiación de su práctica, su consolidación como mercado y el posicionamiento nacional e internacional adquirido durante las últimas décadas, ha convertido el cultivo del grano en el elemento identificador de la cultura regional, además de ser el principal renglón de la economía. 
La zona de influencia del aeropuerto de Palestina y especialmente los municipios de Manizales, Chinchiná y Palestina, se reconocen de acuerdo con información de la Federación Nacional de Cafeteros como la de mayor productividad en el país; de 38.000 productores, 17.000 tienen certificación en cafés especiales sostenibles, dándose un proceso de agregación de valor al producto. Por su parte, el sector cafetero es considerado por el Gobierno Nacional como estratégico para el país, por su aporte al desarrollo social de las regiones y a la economía colombiana. Gracias al apoyo de la Federación al desarrollo social y económico, la zona cafetera y en particular, el área de estudio, gozan de altos niveles de calidad de vida que superan los promedios nacionales; adicionalmente la subregión es la que ofrece una mayor dinámica y posicionamiento dentro del departamento, pues cuenta con las mejores condiciones en cuanto a oferta de infraestructuras y servicios de todo tipo e índices de calidad de vida.

Este contexto de desarrollo social y económico, unido a la localización en el territorio nacional como a sus valores ambientales, paisajísticos y culturales, le otorga a la región condiciones de competitividad para ser aprovechadas en el marco de la construcción de propuestas que superen los intereses locales.

Como valores agregados, la existencia de una cultura cafetera consolidada y reconocida mundialmente, se constituye en fortaleza y factor de competitividad regional, nacional e internacional, que favorece el aprovechamiento en diversas formas de beneficio socioeconómico, como son las alternativas de producción, además de nuevos productos derivados, agroindustria, tecnologías y conocimiento, aprovechamiento del turismo (paisaje, costumbres, prácticas productivas, etc.) y cultura (capital humano, manifestaciones artísticas, arquitectura, gastronomía, fiestas populares, etc.), entre otras.

Según trabajos realizados por La Comisión Económica para América Latina, el Departamento Nacional de Planeación y la Gobernación de Caldas, las ventajas y fortalezas con que cuenta el departamento de Caldas se concentran en la subregión Centro-Sur; entre ellas se tienen las siguientes: alta concentración de capital humano, de urbanización, fortalezas para integrarse a la economía global, en agroindustria, en salud, en educación y generación de conocimiento, para el turismo, en industria, en metalmecánica, en minería, ventajas comparativas en desarrollo tecnológico e investigación, un territorio variado biodiverso y con alto valor geoestratégico, con apreciables valores visuales y paisajísticos, con un sistema urbano 
multipolar integrado y significativo desarrollo de infraestructura vial y de servicios. Aunque son evidentes algunas desventajas como los elevados índices de pobreza en Neira y Palestina, la alta vulnerabilidad del medio natural y de las infraestructuras físicas, la fragmentación ecosistémica, las amenazas por avalancha e inundación, los desequilibrios en la ocupación del territorio, el déficit de vivienda y espacio público, la alta concentración en la oferta de servicios y una estructura administrativa inadecuada para la gestión del territorio.

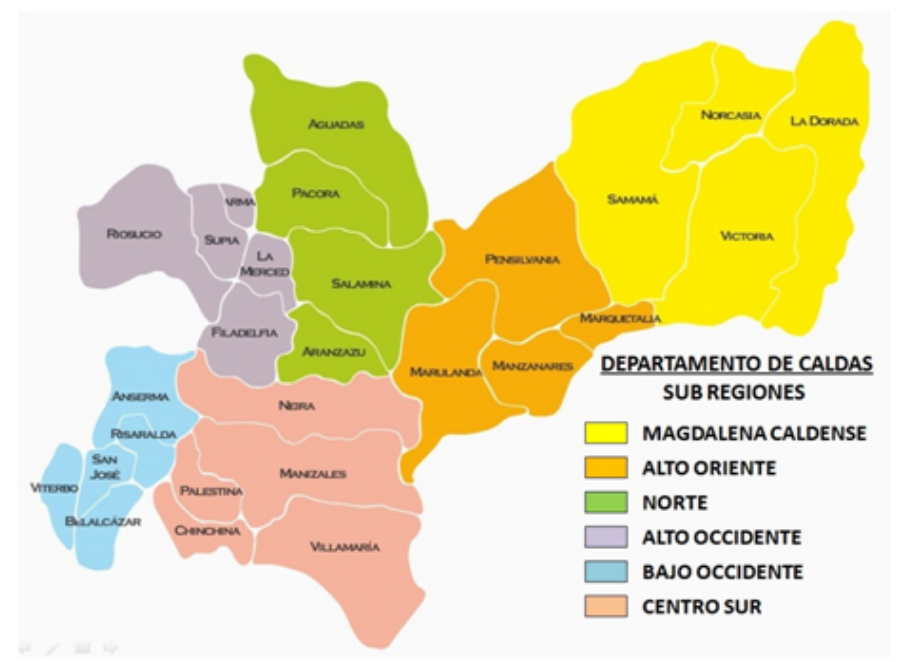

Fuente: Gobernación de Caldas.

Como resultado de la investigación se establecieron los escenarios prospectivos posibles para Palestina, enmarcados en la subregión, desde la espacialización de los determinantes de ordenamiento territorial y su confrontación con las perspectivas de desarrollo municipales; además los escenarios prospectivos propuestos, considerando las oportunidades, posibilidades y limitantes que genera el establecimiento de los macroproyectos. Por último se establecieron las líneas de acción derivadas de los escenarios prospectivos y la construcción de los macroproyectos con incidencia subregional 


\section{MÉTODOS}

Para obtener una visión futura, tomando como base la plataforma natural de la región se han de realizar tres actividades: La primera es el diagnóstico conformado por el inventario y la evaluación de la información recolectada a través diferentes técnicas, dependiendo del grado de información que se tiene; la aplicación de distintas técnicas, se constituye en la evaluación ambiental de la región, para ello se utilizaron la observación, la encuesta y la superposición de mapas. Respecto a la investigación bibliográfica destinada a obtener información de fuentes secundarias se utilizó el análisis de documentos.

La segunda es la prospectiva y muestra los distintos escenarios, que van desde los conflictos de uso, las restricciones y amenazas, sumadas a la orientación que se quiere dar al territorio, para llegar a una tercera etapa que es la propuesta de uso sugerido, con los usos compatibles.

La fase prospectiva del proceso se complementó con un ejercicio SIG, consistente en la superposición de mapas temáticos de usos del suelo, coberturas, vías, centros poblados, pendientes, geología, red hídrica y geomorfología.

La determinación del modelo futuro del territorio representa la fase prospectiva del proceso; la plataforma natural y lo construido son los dos grandes componentes que orientan la construcción de la visión futura.

\section{RESULTADOS}

Uno de los objetivos de la investigación fue ofrecer una metodología práctica para el desarrollo de un proyecto SIG de integración territorial en el municipio de PalestinaCaldas, incluyendo la creación y corrección de la base de datos, el análisis y la presentación de los resultados. La base cartográfica consistió en una serie de mapas temáticos cuyo origen fue el PBOT del municipio de Palestina, Corpocaldas y el Sistema de Información Regional.

Se propuso la búsqueda de emplazamientos idóneos para la construcción de infraestructura, equipamientos y urbanizaciones que darán cuerpo al nuevo entorno municipal de Palestina, ante el agotamiento del suelo de expansión en el centro histórico y las restricciones aeronáuticas que impiden nuevos desarrollos en este lugar. Para lograr dicho objetivo, se planteó una búsqueda temática mediante análisis local, reclasificación y 
superposición de mapas temáticos que tienen en cuenta distintos criterios físico-espaciales, ambientales y determinantes de ordenamiento territorial.

El plano que resume los criterios idóneos para el emplazamiento de Palestina es el siguiente:

Plano número 1: Modelo de Ocupación del Territorio Palestina.

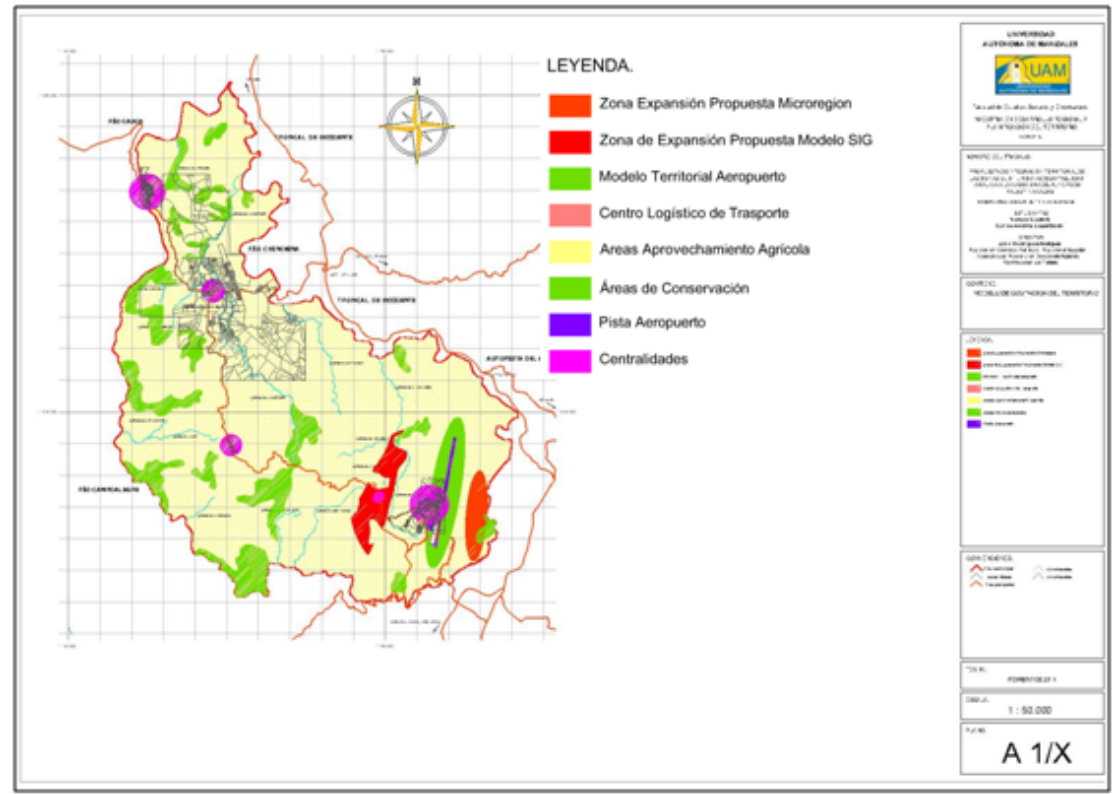

Fuente: Plano base PBOT Palestina, adaptado por los autores

El desarrollo depende de componentes cruciales como la calidad de las instituciones, el cuidadoso diseño y aplicación de políticas económicas, la apertura comercial, la planificación del desarrollo sostenible, la regulación económica, la seguridad para la inversión privada, el acceso al financiamiento, el desarrollo del capital humano, adecuados criterios de evaluación de los proyectos concebidos y la asignación de recursos escasos; sin embargo la infraestructura y la integración de los territorios es una condición necesaria y suficiente para impulsar el desarrollo; de esta manera se hace visible la articulación desarrollo-integración territorial, por lo tanto la promoción del desarrollo lleva implícitas acciones para incorporar física y socialmente los territorios y sus habitantes.

La integración puede ser buscada por los territorios para potenciar sus capacidades con base en el trabajo mancomunado, mejorando la situación de todos sus habitantes. En todos los casos, la integración siempre supone el esfuerzo coordinado, la planeación conjunta y la convivencia pacífica entre los sectores que conforman el 
grupo. Esa es la única forma donde las partes pueden constituir un todo, sin perder su individualidad.

La integración territorial del área de análisis tiene como objetivo fundamental incorporar física y socialmente sus territorios y habitantes para afianzar el desarrollo local y regional a través de las siguientes acciones: eliminar las fronteras municipales enriqueciendo la identidad local, mejorar la accesibilidad, reducir el aislamiento, tanto en las veredas, en las centralidades como en los barrios que conforman el corregimiento y municipio, desarrollar las nuevas potencialidades y oportunidades que ofrece la construcción y operación del Aeropuerto, del Tren de Occidente y del Plan Vial Centro-Sur, asimismo mitigar sus impactos ambientales, identificar aquellos servicios y equipamientos que requiere proveer la centralidad como cabecera municipal, para mejorar la conectividad con sus corregimientos, transferir la toma de decisiones a los territorios, lograr la participación en los desafíos y beneficios del desarrollo y la competitividad, garantizar a los ciudadanos los derechos básicos, cohesión social, superar la inequidad social, económica y cultural, garantizar el acceso al recurso hídrico y promover el respeto por el medio ambiente.

\section{ESCENARIOS PROSPECTIVOS POSIBLES}

Con base en el estudio denominado formulación del macroproyecto de la subregión centro-sur del departamento de Caldas, aeropuerto de Palestina, realizado por Euroestudios (2008), la construcción de escenarios se inscribe dentro del denominado enfoque de planeación estratégica ${ }^{4}$, dirigido a identificar un proyecto posible y deseable para la subregión y el área de influencia del Aeropuerto del Café. Los escenarios se conciben con el fin de apoyar la toma de decisiones y suponen en dicho contexto su socialización y discusión en un ambiente participativo. Su desarrollo y justificación están sujetos al alcance de los diagnósticos previos y a la disponibilidad de información. Tales escenarios simulan condiciones hipotéticas, pero con un buen margen de probabilidad, viabilidad y realismo.

El planteamiento de la propuesta requiere inicialmente la determinación de los siguientes escenarios posibles de desarrollo en el área de análisis: 


\section{ESCENARIOS PARA LA CONFORMACIÓN DE LA ESTRUCTURA SUBREGIONAL}

Teniendo en cuenta dicha clase de escenarios, existen tres de éstos previstos para la zona, cuyo elemento fundamental es la consideración de la construcción del aeropuerto del Café: "Un escenario tendencial" que se construye desde la espacialización de los determinantes de ordenamiento territorial que en la actualidad orientan los usos del suelo en el área definida como de influencia inmediata, que abarca los suelos urbano, suburbano, de expansión urbana y rural de los municipios de Manizales, Chinchiná y Palestina como el suelo rural de Villamaría y Neira; "un escenario multipolar disperso" en el cual se consideran los núcleos poblados urbanos y rurales, localizados en el área de influencia inmediata del aeropuerto, de conformidad con las funciones y su organización nodal; por último, "un escenario en forma de redes con núcleos compactos" que considera los núcleos principales, urbanos y rurales, y las redes viales, partiendo de reconocer la existencia de un polo de actividad principal en el suelo de expansión de Chinchiná y Manizales; área de gran potencial para el desarrollo de actividades de centro logístico, terminal de transporte de carga, zona franca y de servicios conexos para el desarrollo de la actividad aeroportuaria comercial y de carga.

\section{ESCENARIOS PARA EL DESARROLLO DE LA MICRORREGIÓN}

La microrregión está compuesta por los núcleos urbanos de Chinchiná y Palestina, sus áreas adyacentes y las zonas de expansión, El Rosario en el municipio de Manizales, El Edén, La Troncal y La Doctora en Chinchiná y Fundadores en Palestina.

Los POT y PBOT de Manizales, Chinchiná y Palestina indican la asignación de áreas de expansión en el territorio de la microrregión ya señalada; no obstante, ninguna de ellas es explícita en la incidencia que podría tener el Aeropuerto del Café con relación a sus necesidades de articulación y la demanda de los nuevos usos generados por la actividad aeroportuaria y/o sobre la configuración y estructuración de la microrregión.

Dadas las nuevas condiciones de articulación y localización del aeropuerto, se percibe que esta es la oportunidad para reconvertir ciertos usos y funciones urbanas que por su actual ubicación en los cascos urbanos, resultan obsoletas y anti-funcionales. 


\section{ESCENARIOS PROPUESTOS}

Éstos simulan una forma de ocupación de las áreas de expansión, considerando las oportunidades, posibilidades y limitantes que genera la localización del aeropuerto, el cual se entiende como un nodo de actividad regional, capaz de introducir modificaciones en las actuales dinámicas de ocupación y uso del suelo en sus áreas de influencia.

Una de las premisas fundamentales del modelo territorial propuesto, desde el punto de vista de la sostenibilidad ambiental, se basa en las ventajas de los desarrollos compactos, en contraposición del modelo de ciudad difusa, por lo cual se adopta el escenario multipolar disperso como eje de la propuesta; éste se articula en torno a un conjunto de centralidades jerárquicamente dispuestas, evitando la ocupación informal de bordes viales y la urbanización suburbana dispersa.

De la misma manera que se hizo en la determinación de los escenarios para la conformación de la estructura subregional, se han establecido los escenarios prospectivos posibles para la microrregión (Euroestudios, 2008), encontrándose tres (3) alternativas:

Centralidades concentradas. Las áreas residenciales se ubican al norte y sur de las áreas de expansión, concentrando el desarrollo industrial en el centro. Los accesos se realizan a través de la Autopista del Café.

Corredor verde central. Éste escenario se estructura en torno a un corredor de aislamiento verde central que separa las áreas residenciales de las actividades de industria y servicios. Dadas tales circunstancias se requiere de la redistribución de las centralidades en pequeñas unidades que sirven a cada uno de los núcleos resultantes de manera autónoma.

Eje central articulador. Se introduce un corredor vial nuevo que actúa como articulador de los flujos locales e intermunicipales de la zona en expansión intervenida. Se conciben además centralidades auxiliares, generándose un sistema poli-nucleado donde se prevén usos habitacionales industriales y agroindustriales. Este es el escenario que genera mejores posibilidades para Palestina y al que adosamos nuestra propuesta de integración territorial.

Palestina posee terrenos adyacentes a las áreas de expansión de Manizales y Chinchiná, objeto de este ejercicio prospectivo, pero no fueron consideradas en el 
PBOT municipal como tales; sin embargo en razón de su importancia estratégica, dichas zonas deben ser declaradas como de expansión en la revisión y actualización del PBOT e integradas a los escenarios propuestos para el desarrollo de la microrregión. Tales áreas fueron determinadas a través del ejercicio Sig.

Además este escenario podría ser fácilmente articulado a las centralidades concebidas para dar solución a la reubicación del municipio de Neira, aquejado por graves problemas de riesgo, pero también de Villamaría, el cual está en vía de agotar sus posibilidades de expansión y que potencialmente podría verse afectado gravemente por deslaves y avalanchas que discurren a través del río Chinchiná y sus tributarios, desde el Parque Natural Los Nevados.

Para el municipio de Neira se proyectan áreas localizadas en el kilómetro 41, sitio geoestratégico de acuerdo con los nuevos desarrollos subregionales; para Villamaría en el sector Nueva Primavera, próximo a Chinchiná y Palestina, debido a sus dinámicas y potencialidades. La articulación de la micro-región con estas tendencias de localización de Neira y Villamaría se realizaría a través de la Autopista del Café y la Troncal de Occidente, situación que de concretarse elevaría el escenario micro-regional a la condición de subregional.

Plano número 2: Escenario propuesto para la subregión con articulación de sus zonas de expansión

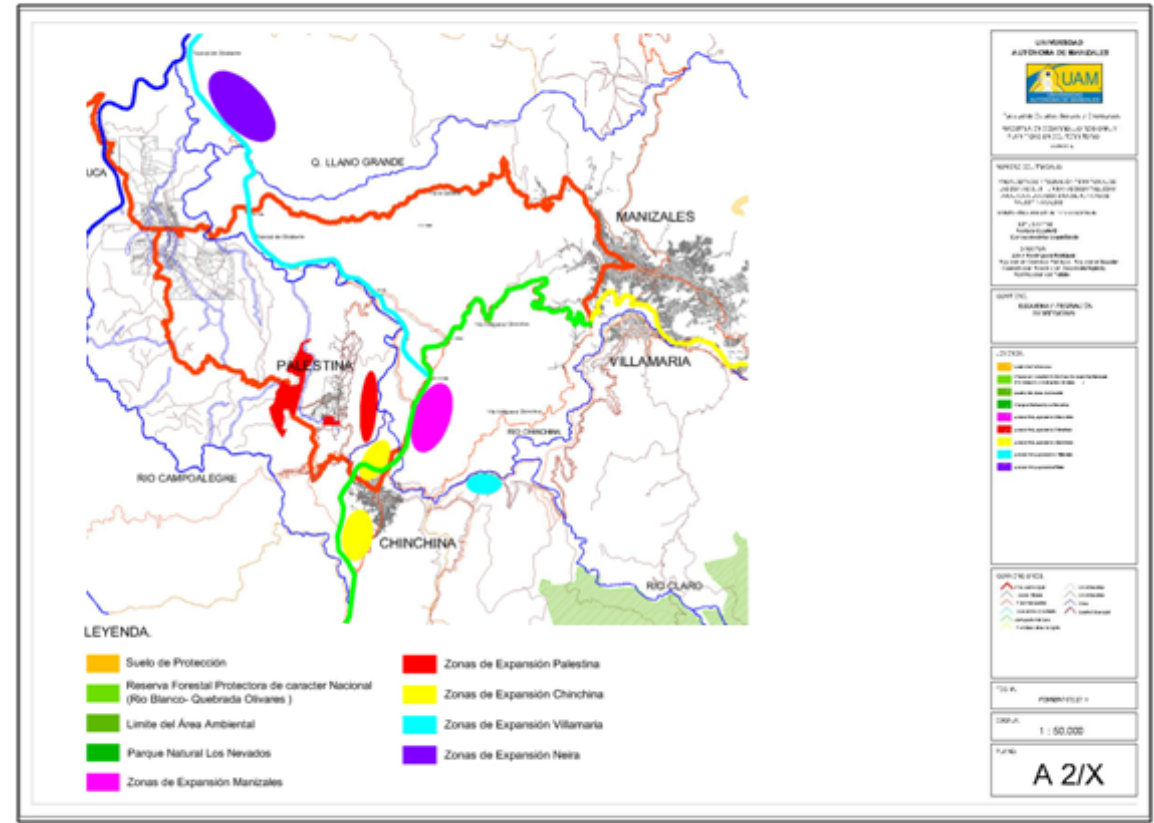

Fuente: Plano base Corpocaldas, adaptado por los autores 


\section{ESCENARIOS PARA EL DESARROLLO DEL AEROPUERTO Y SU ÁREA DE INFLUENCIA}

Las oportunidades y potencialidades regionales que abre esta nueva infraestructura se asocian en parte con la longitud de la pista para atender aeronaves de mayores especificaciones y rango de vuelo.

La construcción de una pista de mayor tamaño incrementa los impactos, aunque no necesariamente de manera exponencial, ya que la mayoría han sido detectados, evaluados, siendo objeto de propuestas y medidas de manejo en el Estudio de Impacto Ambiental para la pista actualmente licenciada $(2.700 \mathrm{~m})^{5}$. Desde el punto de vista de la capacidad aeroportuaria instalada, el tamaño de la pista es significativo con relación al desarrollo regional y las dinámicas territoriales que desencadene. Los escenarios de $2.700 \mathrm{~m}$. y $3.600 \mathrm{~m}$. son los que evidentemente abren nuevas oportunidades y posibilidades de desarrollo regional en un contexto de apertura de mercados.

La construcción del aeropuerto de las especificaciones concebidas finalmente no basta para aprovechar sus potencialidades; es necesaria la realización de ambiciosos acuerdos de integración regional, la racionalización de la oferta aeroportuaria en la región y el desarrollo de infraestructura vial además de servicios asociados al transporte.

\section{ESCENARIOS POSIBLES DE GESTIÓN}

Como escenarios de gestión para los municipios de la subregión se tienen la conformación del Comité de Integración Territorial (CIT), el área metropolitana centrosur y la asociación de municipios.

Líneas de acción derivadas de los escenarios prospectivos y la construcción de macroproyectos. Esta propuesta se fundamenta en el estudio denominado formulación del macro-proyecto de la subregión Centro-Sur del departamento de Caldas, Aeropuerto de Palestina (Euroestudios, 2008); sobre esta base conceptual realizamos ajustes que contribuyen a la consolidación de las estructuras subregionales municipal y micro regionales y así: 
Para la conformación de la estructura subregional. La tradicional visión endogámica de los municipios de la subregión requiere ser revisada y transformada en una visión de más amplia escala que promueva el desarrollo endógeno sostenible y equitativo, lo que implica entender cada unidad territorial como un componente de orden mayor, asimismo parte integral del sistema de ciudades y núcleos urbanos que la componen.

Desde esta perspectiva la localización de funciones regionales y urbanas deberá atender los principios constitucionales de coordinación, concurrencia y subsidiaridad, en particular aplicados en los siguientes temas:

- Armonización en los POT y PBOT de los criterios y prioridades de manejo de las cuencas y micro-cuencas regionales, en particular para la reforestación coordinada de rondas, el tratamiento de aguas servidas y la designación de usos del suelo compatibles en áreas adyacentes. Deben articularse las revisiones a los Planes de Ordenación y Manejo de las Cuencas de los ríos Chinchiná y Campo Alegre.

- Reducción de la vulnerabilidad sísmica y por deslizamiento en áreas propensas a riesgo.

- Conformación del nodo de integración regional con base en los macroproyectos Aeropuerto del Café, Tren de Occidente y el desarrollo de actividades complementarias asociadas en los municipios de Manizales, Chinchiná y Palestina.

- Consolidación de los subsistemas urbanos de ManizalesVillamaría y Chinchiná-Palestina, este último enmarcado en el nodo de integración regional.

- La reducción significativa de los déficits de vivienda, servicios y conectividad del casco urbano de Neira y el mejoramiento de su articulación con Manizales y el aeropuerto a través de la Troncal de Occidente.

- La articulación y utilización de las áreas con potencialidad de desarrollo agrícola en los municipios de Villamaría y Neira; su conexión con lasubregión y el aeropuerto.

- El fortalecimiento de la "Autopista del Café" como eje de integración regional.

- La conformación de los circuitos turísticos y eco-turísticos de la subregión articulados al aeropuerto y a los departamentos de Risaralda y Quindío.

- Fomentar los desarrollos compactos adoptando el modelo territorial basado en el escenario de desarrollo multipolar.

- Reequilibrar las funciones regionales ante la exagerada concentración de funciones en el casco urbano de Manizales y la posibilidad de crear el nodo de integración regional articulado al desarrollo aeroportuario y a las áreas de expansión de los Municipios de Manizales Chinchiná y Palestina; se abre una oportunidad para desconcentrar 
algunas funciones y servicios regionales; se recomienda mejorar la autosuficiencia funcional en los asentamientos rurales y la accesibilidad de éstos con sus respectivas cabeceras; asimismo se busca desconcentrar y ampliar la oferta de empleo en ramas como la agroindustria, los servicios profesionales y financieros además de, la racionalización en los sistemas de transporte terrestre.

- Revisar los usos del suelo rural, lo que implica el examen actualizado con base en la determinación de su capacidad y potencial, para promover aquellos usos más adecuados en función de la naturaleza de los suelos, el equilibrio entre la oferta y demanda ambiental, también la disponibilidad de infraestructuras.

- Afianzar los vínculos terrestres, la conectividad vial interna y externa, pues si bien la subregión exhibe una importante densidad vial, esto no significa que presente condiciones óptimas de articulación interna y externa, máxime frente a las crecientes amenazas por el cambio climático, las condiciones topográficas y de los suelos, la precariedad de sus trazados y diseños y el incremento de los flujos de transporte regionales.

No obstante, los proyectos de ampliación o mejoramiento de la malla vial nacional y proyectos como el Túnel de la Línea o las actuales adecuaciones de las vías de acceso nacionales al puerto de Buenaventura, entre otros, podrían significar una merma en los flujos regionales y una reducción de la importancia de la subregión como articuladora de la Región Central con la Occidental. Las decisiones de política orientadas a la diversificación de la actividad económica en la subregión, la promoción de su desarrollo endógeno, la mayor movilidad en el eje Medellín-Cali-Buenaventura y la construcción y operación del Aeropuerto del Café y del Tren de Occidente, reclaman el mejoramiento de la actual malla vial regional además de una mejor articulación en la misma.

Es claro que la consolidación del sistema de ciudades sólo es posible si se soporta en una adecuada malla vial, lo cual sugiere que el desarrollo de la infraestructura vial y de transporte sea considerado como una prioridad en las acciones de desarrollo territorial para la subregión.

Una visión del papel que la infraestructura, el transporte y los servicios juegan en el desarrollo de la competitividad y la productividad, resulta fundamental para proponer políticas públicas vinculadas con el desarrollo productivo. En particular, los costos de provisión, calidad de los servicios de utilidad pública y transporte son altamente relevantes para la productividad, el crecimiento del PIB y la competitividad de los países, asimismo para el desarrollo y la integración económica. 
Para el desarrollo territorial de la microrregión. Pese a estar conformada por varios municipios contiguos, la microrregión es entendida como una unidad territorial indisoluble. Tal unidad deriva sus perspectivas de desarrollo territorial de la disponibilidad de suelo para los distintos usos asociados con la construcción y la operación del Aeropuerto del Café y del Tren de Occidente, de su actual oferta de servicios, de su infraestructura como de la intención expresa de conformar el nodo de integración regional en dicha área. Las siguientes son las recomendaciones para el manejo de la misma:

Precisar con claridad las especificaciones del aeropuerto. El desarrollo de las áreas de expansión de los tres municipios que conforman la microrregión es posible, si se articula con las oportunidades y necesidades de conexión como de oferta de uso, lo que demanda o induce la construcción y operación del aeropuerto, en cualquiera de sus dimensiones y especificaciones.

No obstante, se aclara que el tipo de aeropuerto que se decida construir es un factor que regulará la demanda de suelo requerido y su dinámica de ocupación. En consecuencia es indispensable que se haga la precisión requerida para ajustar el desarrollo de la microrregión con base en el tipo y especificaciones del equipamiento aeroportuario.

Aprovechamiento de las nuevas ventajas de comunicación del aeropuerto. La construcción y operación del aeropuerto está asociada al mejoramiento de la accesibilidad y conectividad vial como a la ampliación de la oferta de transporte; ella introduce cambios en las actuales dinámicas de urbanización y crecimiento de los cascos urbanos de Chinchiná y Palestina, generando demanda de vivienda y de sus servicios complementarios, de superficies industriales, comerciales, de bodegaje y de servicios de apoyo al transporte, cargue, descargue, parqueos, etc. Finalmente abre la posibilidad de nuevos desarrollos turísticos o eco-turísticos vinculados a la actual oferta en el corregimiento de Santágueda. Para ello se requiere que el nodo de integración regional se planee con base en los siguientes parámetros:

- Conformación de los accesos y conexiones de las áreas de expansión.

- Construcción y adecuación de las conexiones viales al aeropuerto.

- Desarrollo de los planes parciales para las áreas de expansión. 
Desarrollos mixtos y autosuficientes. Los nuevos desarrollos deberán concebirse de manera integral con un único plan parcial por cada área, pero su ejecución podrá ser prevista por fases, en función de la demanda, con el fin de reducir inversiones ociosas y factores de riesgo e incertidumbre en el inversionista.

Para el desarrollo por fases será necesario hacer las previsiones de suelo requeridas para alojar los equipamientos y los servicios, comenzando por aquellos de mayor escala, a fin de que en el futuro no se generen déficits de tierras o resulte muy costosa su adquisición.

Se requiere identificar en cada plan parcial las unidades o fases de construcción por etapas, asegurando que cada una de ellas incluya los servicios y los equipamientos requeridos para apoyar la actividad y reducir la presión de la demanda generada en las infraestructuras y equipamientos circunvecinos.

Oportunidad de reconversión de usos y solución de déficits. El desarrollo de la microrregión deberá ser abordado en una dimensión más amplia que la exclusiva respuesta a las oportunidades y ventajas del nuevo aeropuerto, del Tren de Occidente y Plan Vial Centro-Sur. Su principal objeto es introducir elementos de racionalidad en la distribución de las actividades y funciones de los núcleos urbanos; favorecer su consolidación y desarrollo equilibrado y sostenible además de, contribuir a solucionar los déficits acumulados.

El nuevo desarrollo urbanístico abre la oportunidad para el traslado de industrias dispersas en el casco urbano de Manizales, convertidos en "enclaves" dentro de áreas residenciales, que históricamente han afrontado dificultades de acceso y transporte por la topografía y la configuración de la malla vial. Las áreas vacantes podrán ser tratadas mediante procesos de renovación o re-desarrollo con la incorporación de viviendas y actividad terciaria, según aplique.

También deben concebirse para reducir los actuales déficits municipales de vivienda social y equipamientos de escala urbana (caso escenarios deportivos de Chinchiná), con la ventaja de que ello favorece la integración de los nuevos desarrollos con los núcleos urbanos circunvecinos evitando así la segregación socio-espacial de sus habitantes.

Con relación a la vivienda, el nuevo suelo que se genere, sea por los nuevos desarrollos o por la reconversión de 
uso, deberá otorgar prioridad a la relocalización de los asentamientos ubicados en áreas de riesgo no mitigable y el tratamiento de estas áreas mediante obras de estabilización y contención, asociadas a la reforestación y adecuación o construcción de parques en las áreas tratadas.

Para el desarrollo del Aeropuerto y su área de influencia. El área para el desarrollo del macroproyecto corresponde fundamentalmente al casco urbano de Palestina, las construcciones y adecuaciones aeroportuarias y sus alrededores. Se debe prioritariamente redefinir el perímetro urbano de Palestina y los usos del suelo en áreas adyacentes al aeropuerto teniendo en cuenta que existen predios o porciones del mismo, clasificados como suelo rural. En consecuencia se requiere para efectos catastrales y fiscales, la actualización catastral y la redefinición del perímetro actual de los predios, para incorporar dentro de la clasificación de suelo urbano a la totalidad de los dominios que conformarán el equipamiento aeroportuario.

Dadas las restricciones aeroportuarias y las conclusiones del estudio de impacto ambiental, se requiere de una reconversión de uso en las áreas cubiertas por la franja de ruido " $C$ ". Adicionalmente se requiere la designación de áreas para uso mixto en los sectores de Fundadores, Alto Bonito y Cartagena, para permitir la localización de los nuevos equipamientos urbanos, conjuntamente con la evaluación permanente de los resultados de la ejecución del plan de manejo ambiental del proyecto, de tal manera que puedan atenderse adecuadamente sus impactos ambientales previstos y residuales, dando especial atención a la reconstrucción de los tejidos sociales de la población afectada.

Desarrollo del plan vial municipal. La construcción y adecuación del aeropuerto implica la transformación de la malla vial municipal y de los actuales accesos al casco urbano, en particular para relacionarlo con sus áreas rurales circunvecinas.

El nuevo plan vial deberá abordar los siguientes requerimientos de acceso y circulación:

- Asegurar la conexión del casco urbano con sus áreas rurales circunvecinas, y corregimientos (Santágueda, Arauca, La Plata) y con el municipio de Chinchiná.

- Proveer acceso fluido y seguro hacia la nueva terminal de transporte público. 
- Garantizar la conectividad y acceso del centro tradicional con el nuevo desarrollo del área de Fundadores, con Alto Bonito y Cartagena.

- Garantizar la conexión y acceso de la malla vial peatonal en la totalidad del casco urbano.

- Incorporar los requerimientos de acceso y circulación requeridos por la operación del aeropuerto en cualquiera de sus extensiones y/o etapas de construcción previstas.

- Plantear la construcción de un anillo vial alrededor del aeropuerto, que minimice los impactos en la movilidad de los habitantes en el área de influencia inmediata.

Formular y ejecutar los planes parciales. El macroproyecto prevé la formulación de dos (2) importantes planes parciales con la finalidad de armonizar la demanda y requerimientos aeroportuarios con la estructura urbana preexistente. Estos son los planes parciales para la renovación del centro tradicional y del nuevo desarrollo en el área de Fundadores. Adicionalmente debe considerarse un plan parcial de las áreas determinadas a través del ejercicio como del potencial uso urbanístico.

Recuperar las áreas ambientalmente sensibles y relocalizar asentamientos en áreas propensas a riesgo. Las áreas de ladera que constituyen el casco urbano de Palestina están conformadas por una red de pequeños cursos de agua y nacimientos que forman parte de la microcuenca de la Quebrada de Cartagena, tributaria del Río Chinchiná. Esta red está altamente intervenida y contaminada por lo que se hace indispensable su recuperación y saneamiento. En estas áreas es necesario adelantar acciones de restauración ecológica y relocalizar asentamientos de borde, propensos a deslizamientos.

Adoptar el plan de implantación del aeropuerto. La construcción de las instalaciones aeroportuarias con el único propósito de atender la demanda del servicio aeronáutico, no resuelve diversos impactos derivados de su implantación y operación; éstos, en especial los urbanísticos, no han sido planteados en las exigencias licencias y estudios previos.

La actividad aeroportuaria demanda superficies de parqueo, la restitución de las redes peatonales y de servicios fraccionadas, así como del tratamiento adecuado de los espacios públicos adyacentes y corredores de acceso, con el fin de armonizar su integración espacial y funcional con el casco urbano. Es fundamental que se evite la tugurización de sus alrededores que pueda afectar la seguridad aeronáutica, para lo cual es necesario realizar adecuaciones y adaptaciones que contribuyan a prevenir la invasión de tales áreas y a preservar una imagen atractiva 
en su entorno. Es indispensable que la autoridad municipal trace instrucciones específicas al constructor para direccionar la naturaleza y diseño de las intervenciones en las áreas adyacentes, de tal manera que se logre armonizar su integración espacial y funcional. El plan de implantación del aeropuerto debe atender las siguientes prioridades:

- Considerar un programa que atienda el déficit de vivienda del municipio acentuado por la construcción del proyecto.

- Construcción del anillo vial del aeropuerto y la solución de las respectivas intersecciones viales, según las exigencias de las etapas sucesivas de crecimiento de la pista.

- Adecuación de las vías de acceso principales.

- Construcción de las áreas de parqueo de taxis y visitantes, adicionales a las previstas, que son requeridas para evitar la invasión de las vías adyacentes.

- Adecuación del talud adyacente al casco urbano como parque lineal, que incluya redes peatonales, dotaciones recreativas, miradores y paisajismo.

- Adecuación de las áreas de chaflán, que incluye el cerramiento con cercas vivas, la reforestación, las obras de drenaje y su estabilización.

- Intervención de las culatas generadas por la demolición parcial de edificaciones.

- Diseñar estrategias que atraigan inversión para el desarrollo de áreas y servicios de interés comercial y de demanda del aeropuerto (hoteles, bodegas, nodo logístico de transporte).

Para el desarrollo del Plan vial departamental y rehabilitación del tren de occidente. El plan vial de caldas 2008-2019 fue declarado como de importancia estratégica regional por el gobierno Nacional a través del Documento Conpes 3589 del Consejo Nacional de Política Económica y Social y el Departamento Nacional de Planeación.

De acuerdo con este documento (DNP, 2009) y teniendo en cuenta que el sector transporte es una de las principales bases de la economía como vínculo de las actividades productivas, comerciales y sociales, el Gobierno Nacional adelanta y promueve políticas y estrategias que permitan mejorar continuamente la infraestructura vial, como base para dinamizar el desarrollo socio-económico de las regiones del país. En ese sentido, el Plan Nacional de Desarrollo 2006-2010 "Estado Comunitario: Desarrollo para todos", establece en sus bases que el mejoramiento de los sistemas de transporte y de la infraestructura física asociada, son actividades claves para aumentar la competitividad, fomentar la integración regional y promover la prestación de servicios de transporte en términos de calidad, eficacia y eficiencia. 
El Plan Vial de Caldas incluyó la construcción de la doble calzada Chinchiná-Curazao-Cartagena y Tres PuertasSantágueda, de fundamental importancia para la región y su integración.

El Plan pretende pavimentar más de $500 \mathrm{Km}$ de carreteras e incluye el circuito Centro-Sur, que considera el desarrollo vial, turístico, paisajístico, de renovación urbana, ciclo rutas y doble calzada, en la zona de influencia del Aeropuerto del Café, en construcción.

Un componente fundamental de dicho plan es el desarrollo vial de la subregión Centro-Sur de Caldas que involucra a los municipios de Manizales, Villamaría, Chinchiná, Palestina y Neira; lo que permitirá su conexión con el Aeropuerto del Café y el Tren de Occidente. A su vez, posibilitará la articulación de este desarrollo vial a las vías que conectan a Caldas con los departamentos vecinos, específicamente las del Eje Cafetero (Risaralda y Quindío) y con los corredores de comercio exterior, que cumplen una labor primordial en los procesos de producción, comercialización de productos, integración regional y nacional, a través de su conexión con el corredor arterial complementario de competitividad denominado Transversal Cafetera.

Los corredores arteriales para la competitividad fueron identificados en el marco de la Visión Colombia II Centenario y la Agenda Interna para la Productividad y la Competitividad, como prioritarios y fundamentales para contribuir al logro de una mayor competitividad, impacto y productividad regionales.

El sistema vial Centro-Sur de Caldas incluye cinco (5) tramos: Tres Puertas-Alto del Paisa, Palestina-Chinchiná, La Rochela-La Plata-La Parroquia, Alto del Paisa-Arauca y Palestina-La Manuela, los cuales conjuntamente con el anillo vial y las vías de acceso al Aeropuerto del Café, constituirán una red que alimenta a los corredores de comercio que se complementan con el desarrollo del sistema férreo nacional.

El Tren de Occidente tiene su origen en Medellín y contempla diez (10) estaciones entre la Felisa, el puerto de Buenaventura, Ramales a Cartago, La Tebaida y Pereira (Caimalito); con este proyecto se fortalece el corredor del río Cauca y se restablece la conexión Buenaventura-Santa Marta.

Se trata de un proyecto clave para el desarrollo e integración regional en razón que dinamizará el desarrollo 
de otros proyectos estratégicos como los corredores arteriales complementarios de competitividad, el Aeropuerto del Café, el Puerto de Tribugá y la conexión férrea con el Norte y Occidente del país. Las líneas de acción propuestas son:

Articulación modal. El plan vial departamental y el Tren de Occidente deben considerar como línea de acción la integración modal. Estos macroproyectos deben integrarse en un proyecto coherente de transporte multimodal con base en las conexiones de la vía panorama y el puente sobre el río Cauca, de la doble calzada del Café, el ferrocarril y el Aeropuerto del Café en Palestina. Además se consideran dos parques industriales en Chinchiná y el Kilómetro 41, este último adosado a una zona franca. En el POT de Manizales los concibe como un nodo-corredor y área para el desarrollo del conocimiento y la productividad (HTM, 2009).

Articulación al sistema integrado de transporte regional del eje cafetero. Dado que el ordenamiento legal ha desarrollado sistemas integrados de transporte masivo de pasajeros (SITM), se propone que estos sistemas adosados al transporte interurbano entre ciudades capitales, conformen un solo Sistema Integrado de Transporte Regional del Eje Cafetero, aunque se trata de una iniciativa en construcción.

\section{DISCUSIÓN}

Con base en los lineamientos de política y objetivos de desarrollo de Palestina se plantea el modelo de ocupación del territorio propuesto, el cual se orienta a:

- Lograr la articulación e integración del territorio, con el propósito de superar la desarticulación interna y modificar el actual patrón de aislamiento físico espacial, promovido por la precariedad de sus vías y de las relaciones funcionales con Santágueda, especialmente con Arauca.

- Desarrollo competitivo con equidad, a partir de la integración de amplias zonas del territorio a las corrientes de desarrollo subregional, mediante un sistema urbano equilibrado y en red, que propicie la integración de los mercados regionales y de éstos con los mercados nacionales e internacionales.

- El uso y ocupación racional y sostenible del territorio con el objetivo de aprovechar mejor el capital natural al vincularlo a procesos productivos dinámicos.

- Adoptar un enfoque de desarrollo sostenible, local, endógeno y regional, para abordar la gestión económica del territorio. 
La consolidación del sistema de ciudades sólo es posible si se soportan en una adecuada malla vial, lo cual sugiere que el desarrollo de la infraestructura vial y de transporte sea considerado como una prioridad en las acciones de desarrollo territorial para la subregión.

Una visión del papel que la infraestructura, el transporte y los servicios juegan en el desarrollo de la competitividad y la productividad, resulta fundamental para proponer políticas públicas vinculadas con el desarrollo productivo. En particular, los costos de provisión y la calidad de los servicios de utilidad pública como de transporte son altamente relevantes para la productividad, el crecimiento del PIB y la competitividad de los territorios; también para su desarrollo e integración económica.

Esta nueva forma de creación de riqueza se caracteriza por nuevas complementariedades en el proceso productivo, en función de la productividad de los factores, lo que determina, junto con la infraestructura disponible, la localización de las instalaciones productivas.

Los resultados de esta elaboración permiten concluir que antes de la implantación de los macroproyectos Aerocafé y Tren de Occidente en el territorio de Palestina, este municipio tenía limitadas posibilidades de desarrollo económico que se tradujeran en calidad de vida para sus pobladores, de hecho muy golpeados con la crisis cafetera y la congelación de tierras e inmuebles por más de quince (15) años, en razón de la previsible construcción de Aerocafé. Estos macroproyectos cambian sustancialmente las ventajas comparativas de Palestina; Aerocafé se ha convertido en el eje articulador del ordenamiento territorial de la subregión Centro-Sur y su construcción ha influido drásticamente en el medio ambiente local de una manera negativa, pero también positivamente en algunas variables fundamentales: empleo, educación, economía. El Tren de Occidente de la misma manera ofrece opciones de desarrollo que el conjunto de municipios de la región debe aprovechar, con base en las herramientas que este ejercicio concibe y el Plan Vial Centro-Sur es el eslabón modal que articulará dichos proyectos. Tales macroproyectos crean en su conjunto las condiciones necesarias de infraestructura básica y de servicios de desarrollo urbano para que el sector empresarial asuma su papel de dinamizador de la economía local; de esta manera contribuye con la competitividad económica territorial.

\section{FUTURAS LÍNEAS DE INVESTIGACIÓN}


De esta elaboración se pueden derivar pautas de investigación futura como las siguientes:

- Elaboración de políticas públicas dirigidas a la integración y la competitividad, de cara a la globalización.

- Realizar el análisis de la especialización por región, microrregión y municipio.

- Estudios de seguimiento del impacto ambiental, económico y sociocultural de los macroproyectos en los municipios de implantación.

\section{BIBLIOGRAFÍA}

- Alburquerque, F. (1997). Cambio Estructural, Globalización y Desarrollo Local. Cepal/llpes, Naciones unidas. Santiago de Chile.

- Boisier, S. (1977). Teorías y metáforas sobre desarrollo territorial, Boletín del ILPES (30 pp..). Santiago de Chile..

- Boisier, S. (1999). Desarrollo local: ¿de qué estamos hablando? En: Revista Paraguaya de Sociología, Asunción, No104.

- Centro de Estudio Regionales y Cafeteros (2007). Identificación y Sub regionalización de los Sectores Estratégicos de Caldas versión para discusión.

- Colombia, Congreso de la República (2011). Ley 1450, Plan nacional de desarrollo 2011-2014. Bogotá.

- Colombia, Departamento Nacional de Planeación (2007). Agenda interna para la productividad y la competitividad de caldas. Bogotá.

- Colombia, Departamento Nacional de Planeación (2009). Documento Conpes 3589, Importancia estratégica de los proyectos de infraestructura vial regional en el departamento de Caldas. Bogotá.

- Comisión Estudios Para América Latina y el Caribe (2007). Escalafón de la competitividad de los departamentos de Colombia.

- Comisión Estudios Para América Latina y el Caribe (2009). Escalafón de la competitividad de los departamentos en Colombia,

Dese: http://www.cepal.org/publicaciones/xml/4/41124/LCL3311P.pdf.

- Comisión Regional de Competitividad de Caldas (2002). Programa de competitividad de Caldas: Estudio de identificación y subregionalización de los sectores estratégicos de Caldas.

- Corporación Autónoma Regional de Caldas (2010). Estructura ecológica principal en el ordenamiento ambiental del territorio. Gaceta oficial № 021 Abril de 2010. 
- Corporación Autónoma Regional de Risaralda y otros (2007). Agenda para el desarrollo sostenible de la Ecorregión..

- Departamento de Caldas. Plan de Desarrollo Departamental, Para hacer de Caldas nuestra mejor empresa (2008-2011). Manizales.

- Departamento Nacional de Planeación (2005). Visión Colombia Segundo Centenario: 2019. Bogotá. Doc. Recuperado de www.dnp.gov.co

- Departamento Nacional de Planeación (2006). Plan nacional de Desarrollo. Bogotá D.C.

- Departamento Nacional de Planeación (2007). Agenda interna para la productividad y la competitividad, documento regional Caldas. Bogotá, D.C

- Departamento Nacional de Planeación (2008). Caracterización del desarrollo territorial Departamental. Bogotá D.C.

- Euroestudios (2008). Formulación del proyecto de la región Centro-Sur del Departamento de Caldas: Aeropuerto de Palestina

- Gobernación de Caldas (2007). Carta estadística del Departamento de Caldas.

- Gobernación de Caldas (2008). Plan de Desarrollo 20082011.

- Hábitat Territorio Medio Ambiente. (2008) - Estructura Ecológica Principal para Caldas.

- Municipio de Chinchiná (1999). Acuerdo 030 Plan Básico de Ordenamiento Territorial, Chinchiná

- Municipio de Chinchiná (2008). Plan de Desarrollo Municipal 2008-2011, Chinchiná

- Municipio de Manizales (2007). Acuerdo 663, Plan de Ordenamiento Territorial. Manizales.

- Municipio de Palestina (1999). Acuerdo 160 Plan Básico de Ordenamiento Territorial, Palestina.

- Municipio de Palestina (2008). Plan de desarrollo municipal 2008-2011. "Palestina el Municipio de que soñamos y queremos"

- Programa de las Naciones Unidas para el Desarrollo (2011). Informe de desarrollo humano Desde: http://hdr.undp.org/es/

- Programa de las Naciones Unidas para el Desarrollo. (2004). Informe sobre Desarrollo Humano. Mundi-Prensa Libros S.A. Madrid.

- Red de Universidades Públicas del Eje Cafetero y Otros. (2004). Ecorregión eje cafetero: un territorio de oportunidades. Pereira, Segunda edición.

- Red de Universidades Públicas del Eje Cafetero (2006). Agenda para el desarrollo sostenible de la Ecorregión eje cafetero-Colombia 2007-2019 "territorio deoportunidades" Consultadoen 13-11-11 Desde: http://www.almamater.edu.co/sitio/Archivos/Documentos/D ocumentos/00000021.pdf. 
- Red de Universidades Públicas del Eje Cafetero (2010). Guía para la integración del plan de manejo del paisaje cultural cafetero en el ordenamiento territorial. Universidad Tecnológica de Pereira.

- Rodríguez, J. (2008). La ciudad más allá del perímetro urbano-la relación entre lo urbano y lo rural. En: Rodríguez, J. (Comp.), Desarrollo regional y planificación del territoriocuadernos de clase Número 1 (pp. 131-172).Manizales, Colombia: Universidad Autónoma de Manizales.

- Rodríguez, J. (2009b). A propósito de la gestión del desarrollo urbano-regional y el hábitat sostenible en la globalización. En: Rodríguez, J. (Comp.).

- Rodríguez, J. (2011). Una aproximación a los conceptos de territorio, regional y desarrollo regional. Cuadernos de Clase Maestría en Desarrollo regional y Planificación del territorio. Universidad Autónoma de Manizales

- Vázquez-Barquero, A. (1977). ¿Crecimiento endógeno o desarrollo endógeno? En Cuadernos del CLAEH, Número 78-79, Montevideo.

1. El presente artículo es resultado del trabajo investigativo para optar al título de Magíster en Desarrollo Regional y Planificación del Territorio, de la Universidad Autónoma de Manizales, bajo la dirección académica de Jahir Rodríguez Rodríguez, en el marco del Grupo de Investigación DESARROLLO REGIONAL SOSTENIBLE de la Universidad Autónoma de Manizales. La investigación fue calificada con Mención de acuerdo al reglamento de postgrados.

2. Ingeniero Civil, egresado de la Universidad Nacional de Colombia, con especializaciones en Ingeniería Ambiental y Derecho Ambiental, además de Maestría en evaluación de Impacto Ambiental. Actualmente tesista de la Maestría en Desarrollo Regional y Planificación del Territorio de la Universidad Autónoma de Manizales. Gerente y Director de proyectos desde el año 1995 de la firma consultora Medio Ambiente Ingeniería Ltda.

3. Arquitecto y especialista en Diseño Urbano de la Universidad Nacional de Colombia. Tesista de la Maestría en Desarrollo Regional y Planificación del Territorio de la Universidad Autónoma de Manizales. Se desempeña como consultor particular.

4. Ciudad concepto que alude a la progresiva redistribución y expansión por el campo o medio rural de la población urbana o, en su defecto, de las formas de vida urbanas. En definitiva, el término se refiere a la urbanización del medio rural. Son áreas, generalmente en las periferias de las grandes ciudades que van siendo colonizadas por ellas, según modelos urbanísticos en los que suele predominar la baja densidad de vivienda. 
5. Estudio de impacto ambiental Aeropuerto de Palestina elaborado por la Empresa Medio Ambiente Ingeniería Limitada para la obtención de la licencia ambiental del proyecto; Norbey Castro Gil participó en el estudio en calidad de Director. 